\title{
CYCLICALLY MONOTONE LINEAR OPERATORS ${ }^{1}$
}

\author{
ELIAS S. W. SHIU
}

\begin{abstract}
A linear operator on a complex Hilbert space $\mathcal{H}$ is called $n$-cyclically monotone if for each sequence $x_{0}, x_{1}, \ldots, x_{n-1}, x_{n}=x_{0}$ of $n$ elements in $\mathcal{K}, \sum_{j=0}^{n-1} \operatorname{Re}\left(T x_{j}-x_{j+1}\right) \geqslant 0$. We show that $T$ is $n$-cyclically monotone if and only if $|\operatorname{Arg}(T x, x)| \leqslant \pi / n, \forall x \in \mathcal{K}$. If $T_{m}$ and $T_{n}$ are $m$ and $n$-cyclically monotone operators, then the spectrum of the product $T_{m} T_{n}$ lies in the sector $\{z \in \mathrm{C}:|\operatorname{Arg} z| \leqslant \pi / m+\pi / n\}$.
\end{abstract}

1. Introduction. Let $H$ denote a real Hilbert space with inner product $(\cdot, \cdot)$. The following is a simplified version of [1, Theorem 3]: Let $f$ and $f_{1}$ be two continuous (not necessarily linear) functions on $H$, mapping bounded subsets into bounded subsets, such that (i) $f$ is monotone, i.e., $(f(x)-f(y), x-y)$ $\geqslant 0, \forall x, y \in H$, (ii) $f_{1}$ is tricyclically monotone, i.e., $\left(f_{1}(x), x-y\right)+\left(f_{1}(y)\right.$, $y-z)+\left(f_{1}(z), z-x\right) \geqslant 0, \forall x, y, z \in H$. Then $I+f f_{1}$ is a homeomorphism.

This paper is motivated by the theorem above and we shall restrict our discussion to the elements in $\mathscr{B}(\mathcal{H})$, the set of bounded linear operators on a complex Hilbert space $\mathcal{H}$. An operator $T \in \mathscr{G}(\mathcal{H})$ is called $n$-cyclically monotone, $n$ an integer greater than one, if for each sequence $x_{0}, x_{1}$, $x_{2}, \ldots, x_{n-1}, x_{n}=x_{0}$ of $n$ points in $\mathcal{H}, \sum_{j=0}^{n-1} \operatorname{Re}\left(T x_{j}, x_{j}-x_{j+1}\right) \geqslant 0$. A 2-cyclically monotone operator will be called accretive [5, p. 279]. The concept of the cyclically monotone operators was first introduced by R. T. Rockafellar [6]. According to [6], an $n$-cyclically monotone operator should be called monotone of degree $(n-1)$; however, we justify our definition with the following theorem: $T$ is $n$-cyclically monotone if and only if

$$
|\operatorname{Arg}(T x, x)| \leqslant \pi / n, \quad \forall x \in \mathcal{K} .
$$

In the last section of this paper we show that if $T$ is accretive and $T_{1}$ is 3 -cyclically monotone, then for each $\lambda$ in the spectrum of $T T_{1},|\operatorname{Arg} \lambda| \leqslant \pi / 2$ $+\pi / 3$; consequently $I+T T_{1}$ is invertible.

2. Notation and preliminaries. Let $\mathbf{C}, \mathbf{R}$ and $\mathbf{R}^{+}$denote the set of complex, real and nonnegative real numbers, respectively. Let $\Omega, \Omega_{1} \subset \mathbf{C}, \Omega \cdot \Omega_{1}$ $=\left\{z z_{1}: z \in \Omega, z_{1} \in \Omega_{1}\right\} ; \mathrm{Cl}(\Omega)$ denotes the closure and $\mathrm{Co}(\Omega)$ the convex hull of $\Omega$. For $\alpha, \beta \in \mathbf{R}, 0 \leqslant \beta-\alpha \leqslant 2 \pi, \Sigma(\alpha, \beta)$ denotes the closed sector $\{z \in \mathbf{C}: \alpha \leqslant \arg z \leqslant \beta\}$. For $\alpha \in \mathbf{R}, 0 \leqslant \alpha \leqslant \pi, \Sigma(\alpha)$ denotes the symmetric sector $\Sigma(-\alpha, \alpha)$.

Received by the editors November 21, 1975.

AMS (MOS) subject classifications (1970). Primary 47A10; Secondary 46M05, 47B44, 47H05.

Key words and phrases. Cyclically monotone operators, numerical ranges, tensor products, spectra of products.

${ }^{1}$ This work was partially supported by NRC Grant No. A4002.

c American Mathematical Society 1976 
For $T \in \mathscr{B}(\mathcal{H}), \operatorname{Re} T=\left(T+T^{*}\right) / 2$ and $\operatorname{Im} T=\left(T-T^{*}\right) / 2 i ; \sigma(T)$ denotes the spectrum and $W(T)$ the numerical range of $T, W(T)=\{(T x, x)$ : $\|x\|=1\} . T$ is called nonnegative if $W(T) \subset[0, \infty)$. We define $A(T)$ $=\mathrm{Cl}(\{(T x, x)\})$. Since $A(T)=\mathbf{R}^{+} \cdot \mathrm{Cl}(W(T))$ and the numerical range of an operator is convex, either $A(T)=\mathrm{C}$ or $A(T)=\Sigma(\alpha, \beta)$ with $\beta-\alpha \leqslant \pi$. If $\mathcal{H}$ is finite dimensional and $0 \in W(T)$, then $A(T)$ coincides with the angular field introduced in [10].

Lemma 1. Let $T, S \in \mathscr{B}(\mathcal{H})$. If $S$ is invertible, then $A(T)=A\left(S^{*} T S\right)$.

LEMMA 2 [5, VI-§1.2]. Let $T \in \mathscr{B}(\mathcal{H})$ and $\alpha \in[0, \pi / 2)$; then the following three statements are equivalent:

(1) $A(T) \subset \Sigma(\alpha)$;

(2) $|(\operatorname{Im} T x, x)| \leqslant \tan (\alpha)(\operatorname{Re} T x, x), \forall x \in \mathcal{H}$;

(3) $|(\operatorname{Im} T x, y)| \leqslant \tan (\alpha)[(\operatorname{Re} T x, x)(\operatorname{Re} T y, y)]^{1 / 2}, \forall x, y \in \mathcal{K}$.

Furthermore, each of these conditions implies

(4) $\|T x\|^{2} \leqslant(1+\tan (\alpha))^{2}\|\operatorname{Re} T\|(\operatorname{Re} T x, x), \forall x \in \mathcal{H}$.

Let $S(n)$ denote the $n$-by- $n$ backward-shift matrix, i.e., $S(n)=\left(\delta_{i+1, j}\right)_{n \times n}$. Let $R(n)=(I-S(n))^{-1}$, then $R(n)$ is the $n$-by- $n$ matrix with l's on and above the diagonal and 0's below the diagonal.

Lemma 3. $A(R(n))=\Sigma(\pi / 2-\pi /(n+1))$.

Proof. Since $R(n)$ is a real matrix, $A(R(n))=A(I-S(n))$. The result follows if we show that $W(S(n))$ is a disc centered at 0 with radius $\cos (\pi / n+1)$. It is easy to see that $W(S(n))$ is a disc centered at 0 . The numerical radius of $S(n)$ is the spectral radius of $\operatorname{Re} S(n)$. Put $U_{m}(\lambda)$ $=\operatorname{det}\left(2 \lambda-S(m)-S(m)^{*}\right), m=2,3, \ldots$ If we define $U_{0}(\lambda)=1$ and $U_{1}(\lambda)$ $=2 \lambda$, then $U_{m}(\lambda)=2 \lambda U_{m-1}(\lambda)-U_{m-2}(\lambda), m=2,3, \ldots$ We notice that $U_{m}(\lambda)$ satisfies the recurrence relations and initial conditions of the Chebyshev polynomial of the second kind $[9$, p. 128]. Thus

$$
U_{m}(\lambda)=\sin ((m+1) \arccos (\lambda)) / \sin (\arccos (\lambda))
$$

Consequently the numerical radius of $S(n)$ is $\cos (\pi / n+1)$.

Proposition [2]. Let $S, T \in \mathscr{B}(\mathcal{H})$ and let $S \otimes T$ denote the tensor product acting on the product space $\mathcal{H} \otimes \mathcal{H}$. Then $\sigma(S \otimes T)=\sigma(S) \cdot \sigma(T)$.

Corollary. Let $\mathcal{H}_{1}$ and $\mathcal{H}_{2}$ be two Hilbert spaces. For $T_{j} \in \mathscr{G}_{(}\left(\mathcal{H}_{j}\right)$, $j=1,2, \sigma\left(T_{1} \otimes T_{2}\right)=\sigma\left(T_{1}\right) \cdot \sigma\left(T_{2}\right)$.

Lemma 4 [8]. Let $T_{j} \in \mathscr{B}\left(\mathcal{H}_{j}\right)$ be a normal operator, $j=1,2$. Then $\mathrm{Cl}\left(W\left(T_{1} \otimes T_{2}\right)\right)=\mathrm{Cl}\left(\mathrm{Co}\left(W\left(T_{1}\right) \cdot W\left(T_{2}\right)\right)\right)$.

Proof. $T_{1} \otimes T_{2}$ is also normal.

$$
\begin{aligned}
\text { L.H.S. } & =\operatorname{Co}\left(\sigma\left(T_{1} \otimes T_{2}\right)\right) \\
& =\operatorname{Co}\left(\sigma\left(T_{1}\right) \cdot \sigma\left(T_{2}\right)\right) \quad \text { by Corollary } \\
& =\operatorname{Co}\left(\operatorname{Co}\left(\sigma\left(T_{1}\right)\right) \cdot \operatorname{Co}\left(\sigma\left(T_{2}\right)\right)\right) \\
& =\operatorname{Co}\left(\operatorname{Cl}\left(W\left(T_{1}\right)\right) \cdot \operatorname{Cl}\left(W\left(T_{2}\right)\right)\right)=\text { R.H.S. }
\end{aligned}
$$




\section{Characterizations of cyclically monotone linear operators.}

ThEOREM 1. Let $T \in \Re(\mathcal{H})$. The following statements are equivalent.

(1) $T$ is $n$-cyclically monotone.

(2) For every sequence $y_{1}, \ldots, y_{n-1}$ of $(n-1)$ points in $\mathcal{H}$,

$$
\sum_{j=1}^{n-1} \operatorname{Re}\left(T y_{j}, \sum_{k=1}^{j} y_{k}\right) \geqslant 0 .
$$

(3) The operator $R(n-1) \otimes T$ on $\mathbf{C}^{n-1} \otimes \mathcal{H}$ is accretive.

(4) $A(T) \subset \Sigma(\pi / n)$.

PROOF. (1) $\Leftrightarrow(2)$.

$$
\begin{aligned}
\sum_{j=0}^{n-1}\left(T x_{j}, x_{j}-x_{j+1}\right) & =\sum_{j=1}^{n-1}\left(T x_{j}-T x_{j-1}, x_{j}-x_{0}\right) \\
& =\sum_{j=1}^{n-1}\left(T y_{j}, \sum_{k=1}^{j} y_{k}\right), \text { where } y_{k}=x_{k}-x_{k-1} .
\end{aligned}
$$

(2) $\Leftrightarrow(3)$.

$$
\begin{aligned}
& \sum_{j=1}^{n-1}\left(T y_{j}, \sum_{k=1}^{j} y_{k}\right)=\sum_{j=1}^{n-1}\left(y_{j}, \sum_{k=1}^{j} T^{*} y_{k}\right) \\
& =\left(\left[\begin{array}{l}
y_{1} \\
y_{2} \\
\vdots \\
y_{n-1}
\end{array}\right],\left[\begin{array}{l}
T^{*} y_{1} \\
T^{*} y_{1}+T^{*} y_{2} \\
\vdots \\
T^{*} y_{1}+T^{*} y_{2}+\cdots+T^{*} y_{n-1}
\end{array}\right]\right) \\
& =\left(\left[\begin{array}{l}
y_{1} \\
y_{2} \\
\vdots \\
y_{n-1}
\end{array}\right],\left[\begin{array}{lll}
T^{*} & & \\
T^{*} T^{*} & & \bigcirc \\
\vdots & & \ddots \\
T^{*} T^{*} & \ldots & T^{*}
\end{array}\right]\left[\begin{array}{l}
y_{1} \\
y_{2} \\
\vdots \\
y_{n-1}
\end{array}\right]\right) \\
& =\left(R(n-1) \otimes T\left[\begin{array}{l}
y_{1} \\
y_{2} \\
\vdots \\
y_{n-1}
\end{array}\right],\left[\begin{array}{l}
y_{1} \\
y_{2} \\
\vdots \\
y_{n-1}
\end{array}\right]\right) \text {. }
\end{aligned}
$$

(3) $\Leftrightarrow(4)$ is an immediate consequence of Lemma 3 and

Theorem 2. Let $T_{j} \in \mathscr{B}\left(\mathcal{H}_{j}\right)$ and $A\left(T_{j}\right)=\Sigma\left(\alpha_{j}, \beta_{j}\right), j=1,2$. Suppose either (i) $A\left(T_{1} \otimes T_{2}\right) \neq \mathrm{C}$, or (ii) $\left(\beta_{1}-\alpha_{1}\right)+\left(\beta_{2}-\alpha_{2}\right) \leqslant \pi$; then $A\left(T_{1} \otimes T_{2}\right)$ $=\Sigma\left(\alpha_{1}+\alpha_{2}, \beta_{1}+\beta_{2}\right)$. 
Proof. Since it is always true that $W\left(T_{1} \otimes T_{2}\right) \supset W\left(T_{1}\right) \cdot W\left(T_{2}\right)$,

$$
A\left(T_{1} \otimes T_{2}\right) \supset \Sigma\left(\alpha_{1}+\alpha_{2}, \beta_{1}+\beta_{2}\right) \text {. }
$$

Consequently, assumption (i) implies assumption (ii).

To show that $A\left(T_{1} \otimes T_{2}\right) \subset \Sigma\left(\alpha_{1}+\alpha_{2}, \beta_{1}+\beta_{2}\right)$, we need only to establish the case $\alpha_{j}=-\beta_{j}$, i.e., $A\left(T_{j}\right)=\Sigma\left(\beta_{j}\right), j=1,2$. Write $T_{j}=\operatorname{Re} T_{j}+\operatorname{Im} T_{j}$, $j=1,2$. Since $\operatorname{Re} T_{j}$ is nonnegative, it has a nonnegative square root $Q_{j}[5$, Theorem V.3.35(iv)]. Furthermore, if we assume that $\operatorname{Re} T_{j}$ is invertible, then $T_{j}=Q_{j} N_{j} Q_{j}$, where $N_{j}$ is the normal operator $I+i Q_{j}^{-1}\left(\operatorname{Im} T_{j}\right) Q_{j}^{-1}, j=$ 1, 2. Thus $T_{1} \otimes T_{2}=\left(Q_{1} \otimes Q_{2}\right)\left(N_{1} \otimes N_{2}\right)\left(Q_{1} \otimes Q_{2}\right)$.

$$
\begin{aligned}
A\left(T_{1} \otimes T_{2}\right) & =A\left(N_{1} \otimes N_{2}\right) \quad \text { by Lemma } 1 \\
& =\mathbf{R}^{+} \cdot \operatorname{Cl}\left(W\left(N_{1} \otimes N_{2}\right)\right) \\
& =\mathbf{R}^{+} \cdot \operatorname{Cl}\left(\operatorname{Co}\left(W\left(N_{1}\right) W\left(N_{2}\right)\right)\right) \quad \text { by Lemma } 4 \\
& =\Sigma\left(\beta_{1}+\beta_{2}\right) \text { by assumption (ii). }
\end{aligned}
$$

Thus the theorem is proved if both $\operatorname{Re} T_{1}$ and $\operatorname{Re} T_{2}$ are invertible. In general, we have $A\left(\left(T_{1}+\varepsilon\right) \otimes\left(T_{2}+\varepsilon\right)\right) \subset \Sigma\left(\beta_{1}+\beta_{2}\right)$ for each $\varepsilon>0$. $\mathrm{Cl}(W(\cdot))$ is continuous with respect to the uniform operator topology [3, Problem 175]; we let $\varepsilon$ tend to 0 and obtain $A\left(T_{1} \otimes T_{2}\right) \subset \Sigma\left(\beta_{1}+\beta_{2}\right)$.

Theorem 1 answers the conjecture raised in [6, p. 500]. The following corollary is a complex linear operator version of [6, Theorem 1] and [7, Theorem 24.8].

Corollary 1. For $T \in \mathscr{B}(\mathcal{H}), T$ is nonnegative if and only if $T$ is $n$-cyclically monotone, $n=2,3 \ldots$.

REMARKS. Since the concept of an $n$-cyclically monotone operator is in essence a finite dimensional one, Theorem 1 can be rephrased for the cases of unbounded operators or sectorial sesquilinear forms [5, §VI-1.2]. An $n$ cyclically monotone linear operator, if defined on the whole Hilbert space, is necessarily bounded [5, Theorem V.3.4].

4. Spectra of products. In this section we study the spectrum location of the product of two operators.

Theorem 3 [10, Theorem 2], [11, Theorem 1]. Let $S, T \in \mathscr{G}(\mathcal{H})$. If $0 \notin \mathrm{Cl}(W(T))$, then $\{\sigma(S T) \cup \sigma(T S)\} \subset \mathrm{Cl}(W(S)) / \mathrm{Cl}\left(W\left(T^{-1}\right)\right)$.

Proof. We note that the nonzero elements of $\sigma(S T)$ and $\sigma(T S)$ are the same [3, Problem 61], and $0 \in \mathrm{Cl}(W(T))$ if and only if $0 \in \mathrm{Cl}\left(W\left(T^{-1}\right)\right)$. If $0 \in \sigma(S T-\lambda)$, then

$$
\begin{aligned}
0 \in \sigma\left(S-\lambda T^{-1}\right) & \subset \mathrm{Cl}\left(W\left(S-\lambda T^{-1}\right)\right) \\
& \subset \mathrm{Cl}(W(S))-\lambda \cdot \mathrm{Cl}\left(W\left(T^{-1}\right)\right)
\end{aligned}
$$

Thus for an $m$-cyclically monotone operator $S$ and an $n$-cyclically monotone operator $T,\{\sigma(S T) \cup \sigma(T S)\} \subset \Sigma(\pi / m+\pi / n)$ if $0 \notin \mathrm{Cl}(W(S))$ or 
$0 \notin \mathrm{Cl}(W(T))$. We conclude this paper by showing that the last assumption is not necessary.

THEOREM 4 (cf. [4]). Let $S, T \in \mathscr{B}(\mathcal{H})$ with $S$ accretive and $T$ satisfying the condition:

(*) There exists a constant $d>0$ such that $\operatorname{Re}(T x, x) \geqslant d\|T x\|^{2}, \forall x \in \mathcal{H}$. Then $(-\infty, 0) \cap\{\sigma(S T) \cup \sigma(T S)\}=\varnothing$.

Proof. Let $\lambda$ be a point in the approximate point spectrum of $S T$, i.e., there exists a sequence $\left\{x_{n}\right\}$ of unit vectors such that $\left\|(\lambda-S T) x_{n}\right\| \rightarrow 0$. Since $\left(\lambda x_{n}, T x_{n}\right)-\left(S T x_{n}, T x_{n}\right) \rightarrow 0$ and $S$ is accretive, $\lim \inf \operatorname{Re}\left(\lambda x_{n}, T x_{n}\right)$ $=\lim \inf \operatorname{Re}\left(S T x_{n}, T x_{n}\right) \geqslant 0$. If we assume $\lambda<0$, then $\lim \sup \operatorname{Re}\left(x_{n}, T x_{n}\right)$ $\leqslant 0$. By $(*), \operatorname{Re}\left(x_{n}, T x_{n}\right) \geqslant d\left\|T x_{n}\right\|^{2}$; consequently, $\left\|T x_{n}\right\| \rightarrow 0$ and this contradicts $\lambda \neq 0$. Thus the approximate point spectrum of $S T$ has no negative numbers, and therefore the boundary of $\sigma(S T)$ has no negative numbers [3, Problem 63]. Hence $(-\infty, 0) \cap \sigma(S T)=\varnothing$.

For $T \in \mathscr{B}(\mathcal{H})$, if $A(T) \subset \Sigma(\alpha)$ with $\alpha<\pi / 2$, then $T$ satisfies (*) by the last part of Lemma 2 . However, the converse does not hold; the example in $[4$, p. 309] is also valid for the complex case.

Theorem 5. Let $T_{j} \in \mathscr{G}(\mathcal{H})$ with $A\left(T_{j}\right)=\Sigma\left(\alpha_{j}, \beta_{j}\right), j=1,2$. Suppose $\left(\beta_{1}-\alpha_{1}\right)+\left(\beta_{2}-\alpha_{2}\right)<2 \pi ;$ then

$$
\left\{\sigma\left(T_{1} T_{2}\right) \cup \sigma\left(T_{2} T_{1}\right)\right\} \subset \Sigma\left(\alpha_{1}+\alpha_{2}, \beta_{1}+\beta_{2}\right) .
$$

Proof. Consider the operators $e^{i \theta_{j}} T_{j}, j=1,2$; vary the real numbers $\theta_{1}$ and $\theta_{2}$ and apply Theorem 4 .

ACKNOWLEDGEMENT. The author wishes to thank A. L. Rubin for his suggestions.

Added February 1976. Professor R. T. Rockafellar has kindly informed the author that Theorem 1, (i) $\Leftrightarrow$ (iv), was anticipated by E. Asplund in his paper A monotone convergence theorem for sequences of nonlinear mappings, Proc. Sympos. Pure Math., vol. 18, part 1, Amer. Math. Soc., Providence, R. I., 1970, pp. 1-19.

\section{REFERENCES}

1. H. Brezis and F. E. Browder, Maximal monotone operators in nonreflexive Banach spaces and nonlinear integral equations of Hammerstein type, Bull. Amer. Math. Soc. 81 (1975), 82-88.

2. A. Brown and C. Pearcy, Spectra of tensor products of operators, Proc. Amer. Math. Soc. 17 (1966), 162-166. MR 32 \#6218.

3. P. R. Halmos, A Hilbert space problem book, Van Nostrand, Princeton, N. J., 1967. MR 34 \#8178.

4. P. Hess, On nonlinear equations of Hammerstein type in Banach spaces, Proc. Amer. Math. Soc. 30 (1971), 308-312. MR 43 \#7980.

5. T. Kato, Perturbation theory for linear operators, Die Grundlehren der math. Wissenschaften, Band 132, Springer-Verlag, New York, 1966. MR 34 \#3324.

6. R. T. Rockafellar, Characterization of subdifferentials of convex functions, Pacific J. Math. 17 (1966), 497-510. MR 33 \# 1769.

7. , Convex analysis, Princeton Math. Ser., no. 28, Princeton Univ. Press, Princeton, N. J., 1970. MR 43 \#445.

8. T. Saitô, Numerical ranges of tensor products of operators, Tôhoku Math. J. (2) 19 (1967), 98-100. MR 37 \#654. 
9. J. Todd (Editor), Survey of numerical analysis, McGraw-Hill, New York, 1962. MR 24 \# B1271.

10. H. Wielandt, On the eigenvalues of $A+B$ and $A B$, J. Res. Nat. Bur. Standards Sect. B 77B (1973), 61-63. MR 49 \#318.

11. J. P. Williams, Spectra of products and numerical ranges, J. Math. Anal. Appl. 17 (1967), 214-220. MR 34 \#3341.

Department of Mathematics, California Institute of Technology, Pasadena, CaliFORNIA 91125

Department of Mathematics and Astronomy, University of Manitoba, Winnipeg, Manitoba, Canada R3T 2N2 